\title{
Breadth, Depth, and Competition
}

\author{
Heski Bar-Isaac ${ }^{1}$ \\ Stern School of Business, New York University
}

June, 2007

\begin{abstract}
In a spatial model of both horizontal and vertical differentiation where an agent can occupy an interval rather than a point on the Hotelling line, one can examine the trade-off between depth (a narrow high quality position) and breadth (a wide low quality range). In particular, the extent of depth or breadth can be non-monotonic in the strength of competition.
\end{abstract}

Keywords: breadth, depth, specialization, competition, horizontal and vertical differentiation

JEL: D43, J24, J44, L13, L84,

In numerous skilled fields, agents face a trade-off between acquiring broad or specialized skills. Lawyers, consultants, and academics invest in both general widely applicable skills and knowledge, as well as more particular skills and knowledge on subfields, industries, and tasks. Before beginning to practice, for example, a lawyer could choose a broad education in family law, a greater degree concentration in a particular aspect such as divorce law, or instead focus on corporate law in general, or again a particular aspect, such as corporate tax. An agent's decision on how to make such investments depends on her expectations of the returns for such investment. Demand and the nature and extent of competition from rivals determine the returns to investment in broad and deep skills.

Specialization has been a central concern in economics dating back at least to the opening of Smith (1776). However, the literature has focused on technological and scale

\footnotetext{
${ }^{1}$ I thank Alexei Alexandrov, John Asker, Luis Cabral, Ignacio Esponda, Andrea Prat and participants at the Stern Economics summer seminar for helpful comments and stimulating conversation. Contact info: heski@nyu.edu; Department of Economics, Stern School of Business, NYU, 44 West 4th street 7-73, NYC, NY 10012 USA.
} 
effects for specialization. Essentially, focussing on how efficiency considerations determine the extent of specialization and highlighting the role of technology. Here, in addition, I highlight that strategic/competitive effects can play an important role.

This note presents a preliminary analysis of these strategic effects in a spatial model of both vertical and horizontal differentiation. Depth is captured by vertical quality. Breadth is captured by allowing an agent to position herself on an interval rather than a point on the Hotelling line, reflecting that an agent might possess a broad range of expertise. The model thus allows a simple approach to expressing a trade-off between breadth and depth. I show that strategic concerns lead to outcomes that need not correspond to the efficient benchmark. Further. the focus and central result of this paper is that the extent of specialization might be non-monotonic in the extent of competition.

Though numerous extensions can be envisaged and are briefly discussed in the conclusions, in this note I analyse duopoly on a Hotelling line with agents at either end and constrained to choose either a broad or deep strategy, before competing in price. The extent of competition or differentiation is captured by varying the transport costs on the line. A number of effects arise and allow the extent of specialization in the market to vary non-monotonically in the transport cost. Specifically, at very low transport costs, agents soften price competition by competing on the vertical dimension and so one agent is deep and the other broad. As transport costs increase, the agents compete horizontally by both choosing to specialize. As transport costs increase further, another affect dominates: the agents can charge higher prices by getting closer to customers and saving them travel costs; this entails a broad strategy, and so both agents choose breadth.

As far as I am aware, this is among the first analyses of breadth and depth for an agent and strategic effects in a spatial model. ${ }^{2,3}$ However, the separate effects described are familiar from a couple of papers to which this note owes much. Neven and Thisse (1990) argue that competition shifts from the vertical to the horizontal dimension as transport costs increase. Alexandrov (2006) analyses firms choosing intervals on the Hotelling line in a model of purely horizontal competition and shows how the effect that firms seek to get

\footnotetext{
${ }^{2}$ There is a literature that considers multi-product firms competing both vertically and horizontally, reviewed in Section 5 of Mañez and Waterson (2001). However, the focus with respect to multiple product offerings is with regard to different qualities available at different prices and taking horizontal differentiation fixed. Instead here breadth is understood as appealing to a broader horizontal range. More substantively the model is intended to reflect the sale of a single service (for example the time of a lawyer) where the quantity sold can be understood as probability of sale and is sold at a fixed price (the hourly fee). Thus the model and focus here is quite different from the multi-product case.

${ }^{3}$ Chatain and Zemsky (2007) have a similar motivation in highlighting that strategic as well as productive considerations determine the choice to be broad or specialized. The mechanisms and methods are somewhat different inasmuch as they model the post-location game as cooperative and, more substantively, in that they model "breadth" as a central location on a Hotelling line with demand located at the end points.
} 


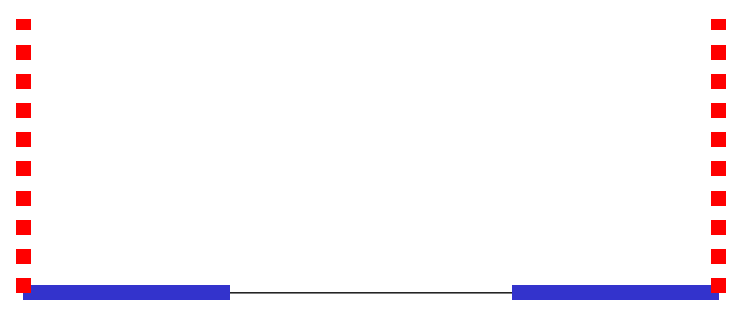

Figure 1: Agents are located at either end of a Hotelling line and choose between broad (blue/solid) and deep (red/dashed) strategies.

close to customers can lead profits to be non-monotonic in transport costs.

Other literature, in particular various interesting works by Garicano and Hubbard (2003, 2006), has examined the extent of specialization in legal markets and focussed primarily on determining how technology and market structure affect the extent of firm specialization and firm organization, but does not allow for non-monotonic effects.

\section{Model}

Two agents are located at either end of a Hotelling line of length 1 . At the first stage of the game, they simultaneously choose either a broad/general strategy, or a deep/specialized strategy. For $L$, the agent on the left of the line, this is a choice $\left(q_{L}, Y_{L}\right) \in\{(0,[0, \alpha]),(1,\{0\})\}$ where the first component $q_{L}$ denotes the vertical quality and the second component $Y_{L}$ the horizontal range. We restrict $\alpha \in\left(0, \frac{1}{2}\right)$. Similarly the right agent $R$ chooses between $(0,[1-\alpha, 1])$ and $(1,\{1\})$.

After observing each other's strategies, the rivals compete in prices $p_{L}$ and $p_{R}$.

A consumer of type $(x, \theta)$ when purchasing from an agent with strategy $\left(q_{i}, Y_{i}\right)$ at a price $p_{i}$ obtains utility

$$
U\left(Y_{i}, q_{i}, p_{i} \mid x, \theta\right)=\bar{U}+\theta q_{i}-p_{i}-t\left(Y_{i}, x\right)
$$

where $t\left(Y_{i}, x\right)=t * \inf \left\{(y-x)^{2}\right.$ s.t. $\left.y \in Y_{i}\right\}$. Consumers are uniformly distributed on a unit square: $x \sim U[0,1]$ and, independently, $\theta \sim U[1,2]$.

Note the timing in the model: The choice of depth or breadth takes place before trade and the agents compete by setting a single price The model is intended to reflect, for example, lawyers' choices of whether to study or train in a broad field, or focus their studies in a particular speciality and then competing in setting hourly rates. 


\section{Equilibrium}

Even in this simple game, a full characterization for all parameters is not trivial as there are different constraints that may bind. In particular, an agent when choosing its price might anticipate that marginal customers are indifferent between buying his good and his rival's or between buying its good and not buying. Indeed there may be marginal customers of both types simultaneously. I characterize both extremes, first supposing that agents are local monopolists and next that all consumers are served.

\subsection{Local monopolists}

First suppose that the left agent is deep and a local monopolist so that indifferent consumers are such that $\bar{U}+\theta-p_{L}-t x^{2}=0$. Then profits are given by ${ }^{4}$

$$
p_{L} \int_{1}^{2} \int_{0}^{\left(\frac{\bar{U}+\theta-p_{L}}{t}\right)^{\frac{1}{2}}} d x d \theta
$$

Attempting to take the first order condition with respect to $p_{L}$ and solving gives an analytically awkward expression; however, it is clear that if the monopolist is to make sales, it must be the case that $p_{L} \leq \bar{U}+2$ and that its volume of sales would be no higher than if it were to charge a price of 0 . It follows that one can construct an upper bound on profits that is decreasing in $t$ and, in particular, that profits are arbitrarily small as $t \longrightarrow \infty$.

Next suppose that the left agent is broad and a local monopolist. The agent can always a charge a price just under $\bar{U}$ and sell to the fraction $\alpha$ of consumers who incur no travel costs, so that its profits are always bounded below by $\alpha \bar{U}$ irrespective of transport costs. It is clear, therefore, that as transport costs rise and competition is softened, eventually both agents will prefer to choose broad strategies.

\section{$2.2 \quad$ Full market coverage}

If $t$ is low enough and $\bar{U}$ is sufficiently high, all consumers buy and marginal consumers are defined by indifference between the two agents. There are three cases to consider, where both agents are deep, both are broad, or there is one of each type.

\subsubsection{Both deep}

Suppose that both agents are deep. A consumer $(x, \theta)$ is indifferent between the two when

$$
\bar{U}+\theta-t x^{2}-p_{L}=\bar{U}+\theta-t(1-x)^{2}-p_{R}
$$

\footnotetext{
${ }^{4}$ Note that in writing this down implicitly I assume that $\bar{U}+\theta-p_{L}-\left.t x^{2}\right|_{\theta=1, x=0} \geq 0$, equivalently that $\bar{U} \geq p_{L}$ and that $\left.\left(\frac{\bar{U}+\theta-p_{L}}{t}\right)^{\frac{1}{2}}\right|_{\theta=2} \leq \frac{1}{2}$ or $\bar{U}+2-p_{L} \leq \frac{t}{2}$. It can be shown that the firm's optimal $p_{L}$ is non-increasing in $t$ and for $t$ large enough this must be true.
} 
Solving for indifferent consumers $x=\frac{1}{2 t}\left(t-p_{L}+p_{R}\right)$ allows us to write down the left agent's profit $\frac{1}{2 t} p_{L}\left(t-p_{L}+p_{R}\right)$. The agent's maximization problem defines a best response function or optimal price as a function of the rival's price $p_{L}\left(p_{R}\right)=\frac{t+p_{R}}{2}$. Solving the analogous problem for the right agent and finding the equilibrium at the intersection of the best response functions, one obtains that $p_{L}=p_{R}=t$ and both agents earn profits of $\frac{t}{2}$.

\subsubsection{Both broad}

A consumer $(x, \theta)$ is indifferent between the two when

$$
\bar{U}-t(x-\alpha)^{2}-p_{L}=\bar{U}-t(1-\alpha-x)^{2}-p_{R}
$$

Again, by solving for the indifferent consumer $x=\frac{1}{2 t-4 t \alpha}\left(t-p_{L}+p_{R}-2 t \alpha\right)$ and taking first order condition for profit maximization one obtains $p_{L}\left(p_{R}\right)=\frac{1}{2} t+\frac{1}{2} p_{R}-t \alpha$. One can then solve for $p_{L}=p_{R}=t(1-2 \alpha)$. Profits here are $\frac{t(1-2 \alpha)}{2}$.

\subsubsection{One broad, one deep}

Suppose that the left agent is deep and the right agent is broad. Indifferent consumers are such that:

$$
\bar{U}+\theta-t x^{2}-p_{L}=\bar{U}-t(1-\alpha-x)^{2}-p_{R}
$$

Indifferent consumers can therefore be defined by $x(\theta)=\frac{\theta-p_{L}+p_{R}+t(1-\alpha)^{2}}{2 t(1-\alpha)}$ so long as this is interior. Suppose that $x(1)$ and $x(2)$ are both interior (it is easy to verify that there are parameters under which this is the case and which are consistent with local monopoly for sufficiently high $t$ ). Then $x(\theta)$ is interior for all $\theta$ and we can write $L$ 's profits as $p_{L} \int_{1}^{2} \int_{0}^{\frac{\theta-p_{L}+p_{R}+t(1-\alpha)^{2}}{2 t(1-\alpha)}} d x d \theta$ and $R^{\prime} s$ profits as $p_{R} \int_{1}^{2} \int_{0}^{1-\frac{\theta-p_{L}+p_{R}+t(1-\alpha)^{2}}{2 t(1-\alpha)}} d x d \theta$.

By taking first order conditions and solving for optimal prices, one can obtain that the maximized profits here are $\Pi_{L}^{D B}=\frac{\left(2 t \alpha^{2}-8 t \alpha+6 t+3\right)^{2}}{72 t(1-\alpha)}$ and $\Pi_{R}^{D B}=\frac{\left(2 t \alpha^{2}+4 t \alpha-6 t+3\right)^{2}}{72 t(1-\alpha)}$.

There will be an equilibrium where one agent chooses to be broad and the other agent chooses to be deep if $\Pi_{L}^{D B}>\frac{t(1-\alpha)}{2}$ (so the left agent prefers to be deep than broad) and $\Pi_{R}^{D B}>\frac{t}{2}$ (so that the right agent prefers to be broad than deep). For example with $\alpha=0.2$, this requires that $t<0.286$.

Note that when $t$ is sufficiently small, efficiency would instead require that both agents should choose to be deep. 


\section{Conclusions}

The analysis above suggests that for sufficiently low transport costs, equilibrium will involve one broad and one deep agent. As transport costs increase, the equilibrium sees both rivals deep. As transport costs continue to increase, eventually both agents would choose to be broad local monopolists. Thus the extent of specialization can be non-monotonic in transport costs.

There are a number of extensions and further considerations that one could easily imagine. First, one might wish for a full characterization at all parameter values, alternative specifications for transport costs, and more general distributions of consumers. Second, a more natural interpretation of increasing competition is reducing a cost of entering the industry and allowing agents to locate on a Salop circle, as in Salop (1979). Indeed, the interpretation of a fall in the transport cost as an increase in competition informally appeals to the notion of increased entry and "closer" firms with a fall in entry costs, though a full characterization of entry in a Salop circle model is complicated inasmuch as equidistant positioning is not a natural assumption with asymmetric firms. ${ }^{5}$ Finally, one might consider agents choosing at some cost to extend their breadth (as Alexandrov (2006) allows for in a purely horizontal model) and to choose their depth at some cost, as is typical in vertical models. These are not trivial extensions and in general spatial models can provide technical challenges; however, one may readily anticipate, that similar considerations to those outlined in this note might arise. In particular, as a specific example, should the cost of entry into the legal profession fall, the analysis suggests that there could be either a greater or smaller proportion of specialized lawyers as a result. In principle one could test for such non-monotonic effects.

\section{References}

[1] A. Alexandrov (2006): "Fat Products," working paper, Kellogg School of Management, Northwestern University.

[2] O. Chatain and P. Zemsky (2007): "The Horizontal Scope of the Firm: Organizational Tradeoffs vs. Buyer-Supplier Relationships," Management Science, Vol. 53, No. 4, April 2007, pp. 550-565.

[3] L. Garicano and T. Hubbard (2003) "Firms' Boundaries and the Division of Labor: Empirical Strategies," Journal of the European Economic Association, April/May 2003, 495-502.

[4] L. Garicano and T. Hubbard (2006) "Specialization, Firms, and Markets: The Di-

\footnotetext{
${ }^{5}$ It is possible to characterize circumstances in which all firms are deep and equidistant, and all firms are broad and equidistant on the Salop circle can be supported as an equilibrium.
} 
vision of Labor Within and Between Law Firms," working paper, Kellogg School of Management, Northwestern University.

[5] Juan A. Mañez and Michael Waterson (2001) "Multiproduct Firms and Product Differentiation: A Survey" Warwick Economic Research Papers No. 594.

[6] D. J. Neven and J.-F. Thisse (1990) "Quality and Variety Competition" in Economic Decision Making: Games, Econometrics and Optimisation, Contributions in Honour of Jacques H. Dreze, eds J.J. Gabszewicz, J.-F. Richard, and L.A. Wolsey, North Holland.

[7] Salop, S. C. (1979): "Monopolistic Competition with Outside Goods," Bell Journal of Economics, 10(1), 141-156.

[8] Smith, A. (1776): An Inquiry into the Nature and Causes of the Wealth of Nations 